\title{
Topological and Metrical Aspects of Normal and Abnormal Grain Growth
}

\author{
Paulo Rangel Rios ${ }^{\mathrm{a} *}$, Martin Eden Glicksman ${ }^{\mathrm{b} *}$ \\ ${ }^{a}$ Universidade Federal Fluminense - UFF, \\ Escola de Engenharia Industrial Metalúrgica de Volta Redonda, \\ Av. dos Trabalhadores, 420, 27255-125 Volta Redonda - RJ, Brazil \\ ${ }^{\mathrm{b}}$ University of Florida, Materials Science \& Engineering Department, \\ 32611-6400 Gainesville - FL, USA
}

Received: March 15, 2007; Revised: September 12, 2007

\begin{abstract}
Grain growth theories normally describe a grain using the concept of 'grain radius'. However, this assumption bypasses all topological information relating to the grain, viz., the number of its faces, edges, etc. This study, by contrast, introduces a new methodology, treating normal and abnormal grain growth in three dimensions in terms of both metrical and topological properties of the grains.
\end{abstract}

Keywords: microstructure, grain growth, abnormal grain growth, polycrystals, soap bubbles

\section{Introduction}

The relationship between grain topology and boundary curvature in polycrystalline networks was recognized early on in research conducted on grain growth and soap bubble. Curvature driven boundary migration obeys the well-known fundamental kinetic equation

$$
\mathrm{G}=-\mathrm{M} \gamma \mathrm{H}
$$

where $\mathrm{G}$ is the boundary velocity, $\gamma$ is the boundary free energy per unit of area, M is the boundary mobility, and H is the boundary's mean curvature. The minus sign is included in Equation 1 because the boundary always moves towards its center of curvature.

Unfortunately, a simple and exact topological expression relating the curvature to the number of faces does not exist for grain growth in three dimensions. Therefore, grain growth theories generally are based on the metrical concept of the equivalent 'grain radius'. The 'grain radius' equals the radius of a sphere with its volume equal to the volume of the polyhedral grain. Theories based on grain radius were developed and provide valuable insights into the evolution of polycrystals ${ }^{1}$. An obvious limitation of this metrical approach is that it overlooks topological information, notably, the number of faces comprising a grain.

Recently, one of the authors (MEG) devised an exact analytic method to represent irregular 3-dimensional networks using an infinite set of regular polyhedra with uniformly curved identical faces. These regular polyhedra define the 'average $\mathrm{N}$-hedra' or $\mathrm{ANHs}^{2}$. Every irregular polyhedron with a given number of faces, $\mathrm{N}$, may be represented topologically by a unique highly-symmetrical polyhedron selected from the set of ANHs. ANHs are constructed in such a manner that they satisfy exactly the average topological constraints imposed by a space-filling network in three dimensions. This approach greatly simplifies the analysis of materials configured as real irregular networks, such as polycrystals, as all the geometric properties of ANHs can be calculated exactly. ANHs may, therefore, be used as 'proxies' for analyzing real irregular network grains, allowing the rigorous treatment of related problems pertaining to such network structures ${ }^{2-7}$.

The representation of grains with ANHs (See Equations 2-4 below) suggests that a grain may shrink or grow by two distinct processes, illustrated in Figure 1: topological and metrical. In the topological process the grain shrinks or grows solely by losing or gaining faces, respectively, and the vertex-to-vertex distance, $\lambda$, remains constant. By contrast, during the metrical process the grain shrinks or grows solely by changing its gauge, $\lambda$, without losing or gaining faces. In Figure 1 one starts with a 6-hedron with a vertex-tovertex distance equal to $\lambda+\Delta \lambda$, shown in the upper right of Figure 1 . This grain can decrease its volume by losing faces to become, say, the 4-hedron on the top left of Figure 1 exhibiting the same vertexto-vertex distance, $\lambda+\Delta \lambda$. This is indicated by the arrow labeled ' $T$ ' for topological process. Another possibility is that the 6-hedron may decrease its volume by just decreasing its vertex-to-vertex distance from its original value, $\lambda+\Delta \lambda$, to a smaller value, $\lambda$. In the metrical process the 6-hedron merely becomes a smaller 6-hedron as shown on lower right-hand side of Figure 1. This process is indicated by an arrow labeled "M" for metrical process. Finally, the grain might shrink by a combination of the two processes: losing faces and also decreasing its vertex-to-vertex distance. This combined process is indicated by the arrow labeled ' $\mathrm{T}+\mathrm{M}$ ' in Figure 1 .

In this paper we introduce a new methodology to treat normal and abnormal grain growth. We use the ANH formalism instead of the assumption of equivalent grain radius. The ANHs are employed here as proxies, or topological representatives, in lieu of the actual space-filling irregular polyhedral grains. Application of ANHs leads to a deeper quantitative understanding of the topological and metrical changes that occur during normal and abnormal grain growth.

\section{Construction and Properties of ANHs}

The construction of ANHs has been described in detail in earlier publications $^{2-7}$. Two ANHs, one with four faces, the 4-hedron, and another with six faces, the 6-hedron, are illustrated in Figure 1.

The curvature, H, area, A, and volume, V, of ANHs may be expressed as a product of a topological factor and a metrical scale factor, or gauge, viz.,

$$
\mathrm{H}(\mathrm{N}, \lambda)=\mathrm{h}(\mathrm{N}) \lambda^{-1}
$$




$$
\begin{aligned}
& \mathrm{A}(\mathrm{N}, \lambda)=\mathrm{a}(\mathrm{N}) \lambda^{2} \\
& \mathrm{~V}(\mathrm{~N}, \lambda)=\mathrm{v}(\mathrm{N}) \lambda^{3}
\end{aligned}
$$

The scale factor or linear gauge, $\lambda$, appearing in Equations 2-4 is chosen here as the distance between nearest neighbor vertices on these regular polyhedra.

Equation 4 shows that the volume of an $\mathrm{ANH}$ is a product function of two single valued functions, $v(N)$ and $\lambda^{3}$. These functions and their correspondent independent variables $\mathrm{N}$ and $\lambda$ are independent of each other. In other words, a variation in the volume can be brought about by independently varying either $\mathrm{N}$ or $\lambda$.

Expressions for topological factors corresponding to the (dimensionless) curvature, $\mathrm{h}$, area, a, and volume, $\mathrm{v}$, are not given here for space reasons. But full details of the structure and mathematical properties of ANHs may be found elsewhere ${ }^{2-5}$.

It is important to mention that the mean curvature, $\mathrm{H}$, is found to be positive for $\mathrm{N} \leq 13$, and negative for $\mathrm{N} \geq 14$. The exact noninteger value of $\mathrm{N}$ at which $\mathrm{H}$ changes sign is $\mathrm{N}_{\mathrm{C}}=13.397332571 \ldots$ We define the ' $\mathrm{N}_{\mathrm{C}}$-hedron' or 'critical ANH', as the ANH exhibiting the critical number, $\mathrm{N}_{\mathrm{C}}$, of flat faces, for which $\mathrm{H}=0$. Thus, all ANHs with $\mathrm{N} \leq 13$ have convex faces and tend to shrink, whereas all ANHs with $\mathrm{N} \geq 14$ have concave faces and tend to grow. Another property of the critical ANH that will be used in what follows is its volume, $\mathrm{V}_{\mathrm{C}}=\mathrm{v}_{\mathrm{C}} \lambda^{3}$, where $\mathrm{v}_{\mathrm{C}}=9.8703795 \ldots$

\section{Topological and Metrical Components of Grain Growth}

Two main assumptions are needed in the analysis. The first is that a polycrystal can be represented by ANHs. The second is that
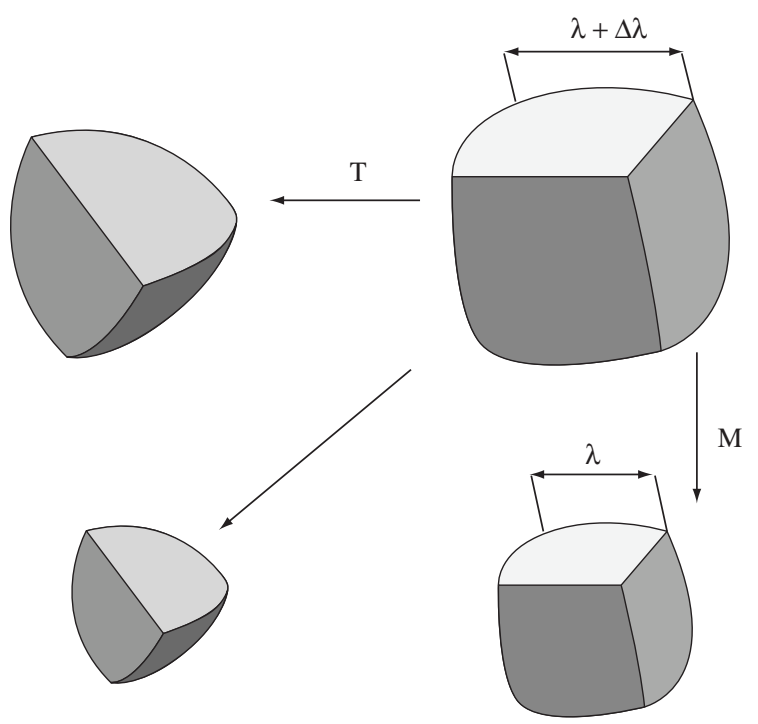

Figure 1. The 6-hedron with a vertex-to-vertex distance equal to $\lambda+\Delta \lambda$, shown in the upper right can decrease its volume by losing faces to become the 4-hedron on the top left exhibiting the same vertex-to-vertex distance, $\lambda+\Delta \lambda$. This is indicated by the arrow labeled ' $\mathrm{T}$ ' for topological process. Another possibility is that the 6-hedron may decrease its volume by just decreasing its vertex-to-vertex distance from its original value, $\lambda+\Delta \lambda$, to a smaller value, $\lambda$. In the metrical process the 6-hedron merely becomes a smaller 6-hedron as shown on lower right-hand side. This process is indicated by an arrow labeled 'M' for metrical process. Finally, 6-hedron might shrink by a combination of the two processes: losing faces and also decreasing its vertex-to-vertex distance. This combined process is indicated by the arrow labeled ' $\mathrm{T}+\mathrm{M}$ '. the linear gauge of each $\mathrm{ANH}$ is, to a first approximation, equal to the average linear gauge of the grain network, $\lambda$.

The implied rate of change in grain volume may be expressed as a total differential of the independent metric and topological variables, namely,

$$
\frac{\mathrm{dV}(\mathrm{N}, \lambda)}{\mathrm{dt}}=\left(\frac{\partial \mathrm{V}}{\partial \mathrm{N}}\right)_{\lambda} \frac{\mathrm{dN}}{\mathrm{dt}}+\left(\frac{\partial \mathrm{V}}{\partial \lambda}\right)_{\mathrm{N}} \frac{\mathrm{d} \lambda}{\mathrm{dt}}
$$

Inserting Equation 4 into Equation 5 one obtains:

$$
\frac{\mathrm{dV}}{\mathrm{d} \tau}=\lambda^{3} \frac{\mathrm{dv}(\mathrm{N})}{\mathrm{dN}} \frac{\mathrm{dN}}{\mathrm{d} \tau}+\frac{3 \lambda}{2} \mathrm{v}(\mathrm{N}) \frac{\mathrm{d} \lambda^{2}}{\mathrm{~d} \tau}
$$

where $\tau=M \gamma t$, is taken as time. The first term on the right-hand side of Equation 6 is the topological contribution to grain growth, whereas the second term is the metrical contribution.

We assume that during grain growth the boundary motion is governed by Equation 1. For our purposes it is convenient to find the rate of volume change from Equation 1 using the well-known Cahn-Hagel equation, $\mathrm{G}=(\mathrm{dV} / \mathrm{dt}) / \mathrm{A}$, one obtains for normal grain growth

$$
\frac{\mathrm{dV}}{\mathrm{d} \tau}=-\mathrm{ha} \lambda
$$

It is assumed that abnormal grains can be represented by ANHs. For abnormal growth in a pinned matrix, matrix grains are pinned by a positive interaction force, $\mathrm{F}>0$, per unit boundary area, which may be written as $F=\gamma Z>0$. Here $Z$ represents some reciprocal length scale, allowing $\mathrm{F}$ to be considered as a 'back pressure' opposing the pressure exerted by the boundary curvature itself. The pinning force, of course, always resists boundary motion, and arises naturally in polycrystals, often as a result of the presence of a fine particle dispersion ${ }^{8}$. The pinning force is normally reported with the total boundary curvature $\left(\kappa_{1}+\kappa_{2}\right)$ whereas in this paper we use the boundary curvature defined as half of this value, $1 / 2\left(\kappa_{1}+\kappa_{2}\right)$. For this reason, in what follows we use $Z / 2$, instead of $Z$, for the pinning force to insure consistency with published papers on this subject. The resultant grain boundary velocity, $\mathrm{G}$, when pinning is present, may be expressed as

$$
\mathrm{G}=\gamma \mathrm{M}(-\mathrm{H}-\mathrm{Z} / 2)
$$

One may solve Equation 8 for the rate of change of abnormal grain volume, $\mathrm{V}_{\mathrm{A}}$

$$
\frac{\mathrm{dV}_{\mathrm{A}}}{\mathrm{d} \tau}=\left(-\mathrm{H}_{\mathrm{A}}-\mathrm{Z} / 2\right) \mathrm{A}_{\mathrm{A}}
$$

where $\mathrm{H}_{\mathrm{A}}$ and $\mathrm{A}_{\mathrm{A}}$ are the grain boundary curvature and area of the abnormal grain, respectively.

This rate laws, Equations 7 and 9 imply that boundary migration remains unhindered by the ensuing series of topological events. In other words, the topological processes are not the controlling, or slow kinetic steps. Topological changes on a grain, such as face creation, switching, and face disappearance, occur as often as the network requires, so that rate of change in volume conforms to the predictions of Equations 7 or 9. This kinetic assumption is implicit in the equivalent grain radius model.

Combining Equations 7 and 9 with Equation 6 allows to obtain differential equations for the change in number of grain faces as a function of time.

For normal grain growth

$$
\frac{\mathrm{dN}}{\mathrm{d} \ln \lambda^{2}}=\frac{1}{\frac{\mathrm{dv}}{\mathrm{dN}}}\left(-\frac{\mathrm{ha}}{\frac{\mathrm{d} \lambda^{2}}{\mathrm{~d} \tau}}-\frac{3}{2} \mathrm{v}\right)
$$


where instead of time $\lambda$ that is a function of time for normal grain growth has been used. The metrical rate remains a constant during self-similar normal grain growth, via the well-known parabolic law, $\mathrm{d} \lambda^{2} / \mathrm{d} \tau=$ constant $>0$.

During abnormal grain growth in a fully pinned matrix, the matrix grains are immobilized by pinning. Therefore the vertex-to-vertex distance between neighboring matrix grains remains fixed during abnormal grain growth, and equal to the length scale, $\lambda$. The metrical rate therefore is equal to zero for abnormal grain growth in pinned matrix, $\mathrm{d} \lambda^{2} / \mathrm{d} \tau=0$. This means that only the topological contribution remains. In other words, in a fully pinned matrix an abnormal grain can only grow by gaining faces. Such a seemingly obvious result has never to our knowledge been pointed out before.

The corresponding differential equation for abnormal grain growth in a fully pinned matrix becomes

$$
\frac{\mathrm{dN}_{\mathrm{A}}}{\mathrm{d} \tau_{\mathrm{A}}}=\frac{\left(-\mathrm{h}_{\mathrm{A}}-\mathrm{Z} \lambda / 2\right) \mathrm{a}_{\mathrm{A}}}{\left(\partial \mathrm{v}_{\mathrm{A}} / \partial \mathrm{N}_{\mathrm{A}}\right)_{\lambda}}
$$

where $\tau_{\mathrm{A}}=\left(\mathrm{M} \gamma / \lambda^{2}\right) \mathrm{t}$. The subscript "A" will be dropped from $\tau_{\mathrm{A}}$ in what follows, for simplicity.

\section{Quantitative Analysis of Topological and Metrical Components of Grain Growth}

In order to separate the two types of contributions to volume change: metrical and topological one must know the value of $\mathrm{d} \lambda^{2} / \mathrm{d} \tau$. Rios and Glicksman ${ }^{7}$ have shown that $\mathrm{d} \lambda^{2} / \mathrm{d} \tau=0.229$. Using this one may return to Equation 6 and segregate the topological and metric contributions to grain volume change. The topological contribution is given by

$\left(\frac{\mathrm{dV}}{\mathrm{d} \tau}\right)_{T}=(-\mathrm{ha}-0.3435 \mathrm{v}) \lambda$

and the metric contribution is given by

$\left(\frac{\mathrm{dV}}{\mathrm{d} \tau}\right)_{\mathrm{M}}=0.3435 \mathrm{v} \lambda$

The total grain volume change is the sum of both contributions, namely, Equation 6.

Each factor is plotted as a function of the number of faces in Figure 2 for $\lambda=1$.

Figure 2 shows that the total grain volume change (See ' $T+$ M - curve' in Figure 2), as expected from our ANH studies, decreases for grains with positive curvature, i.e., grains with less than $\mathrm{N}_{\mathrm{C}}=13.397$ faces, and increases for grains with a greater number of faces. The metric contribution(See ' $M$ - curve' in Figure 2) is always positive. It increases with an increase in the number of grain faces. Grains with a large number of faces tend to have a large metric contribution whereas grains with a small number of faces tend to have a small metric contribution. The topological contribution (See ' $\mathrm{T}$ - curve' in Figure 2) behaves in the opposite way, it is always negative and its absolute value decreases with an increase in the number of grain faces. Close to the cut-off grain, grains grow almost only by an increase in their vertex-to-vertex distance. Conversely, at the lowest number of faces grains tend to decrease their volume almost exclusively by losing faces.

Finally, the trajectory of a certain grain in $(\mathrm{N}, \mathrm{V})$ space can be obtained first, integrating Equation 10

$$
\int_{\mathrm{N}_{0}}^{\mathrm{N}} \frac{1}{\mathrm{dv}}\left(-\frac{\mathrm{ha}}{\mathrm{d} \lambda^{2}}-\frac{3}{2} \mathrm{v}\right) \mathrm{dN}=\ln \left(\frac{\lambda^{2}}{\lambda_{0}^{2}}\right)
$$

where $\mathrm{N}_{0}$ is the number of faces of a certain grain at $\lambda=\lambda_{0}$. Equation 13 is plotted in Figure 3 for $\mathrm{N}_{0}=10,20$ and 30. As expected the number of faces decrease with $\lambda$ irrespective of the grain having $\mathrm{N}$ larger or smaller than $\mathrm{N}_{\mathrm{C}}$.

Inserting Equation 13 into Equation 4 one obtains the grain volume as a function of its number of faces

$$
\mathrm{V}\left(\mathrm{N}, \mathrm{N}_{0}\right)=\lambda_{0}^{3} \exp \left(\frac{3}{2} \int_{\mathrm{N}_{0}}^{\mathrm{N}} \frac{1}{\mathrm{dv}}\left(-\frac{\mathrm{ha}}{\mathrm{dN}}-\frac{3}{\mathrm{~d} \lambda^{2}} \mathrm{v}\right) \mathrm{dN}\right)
$$

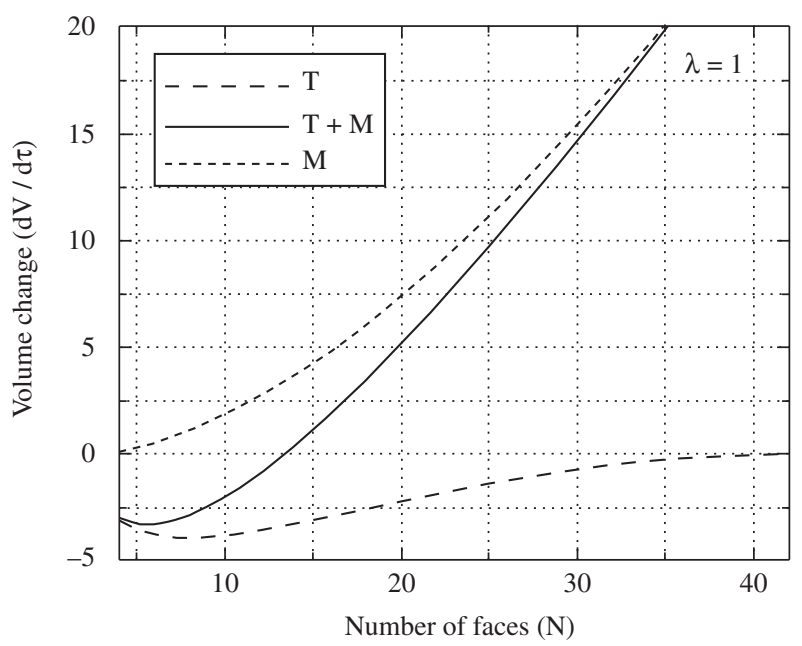

Figure 2. The total grain volume change ' $\mathrm{T}+\mathrm{M}$ - curve' decreases for grains with positive curvature, i.e., grains with less than $\mathrm{N}_{\mathrm{C}}=13.397$ faces, and increases for grains with a greater number of faces. The metric contribution, 'M - curve' is always positive. It increases with an increase in the number of grain faces. Grains with a large number of faces tend to have a large metric contribution whereas grains with a small number of faces tend to have a small metric contribution. The topological contribution, ' $T$ - curve' behaves in the opposite way, it is always negative and it decreases with an increase in the number of grain faces.

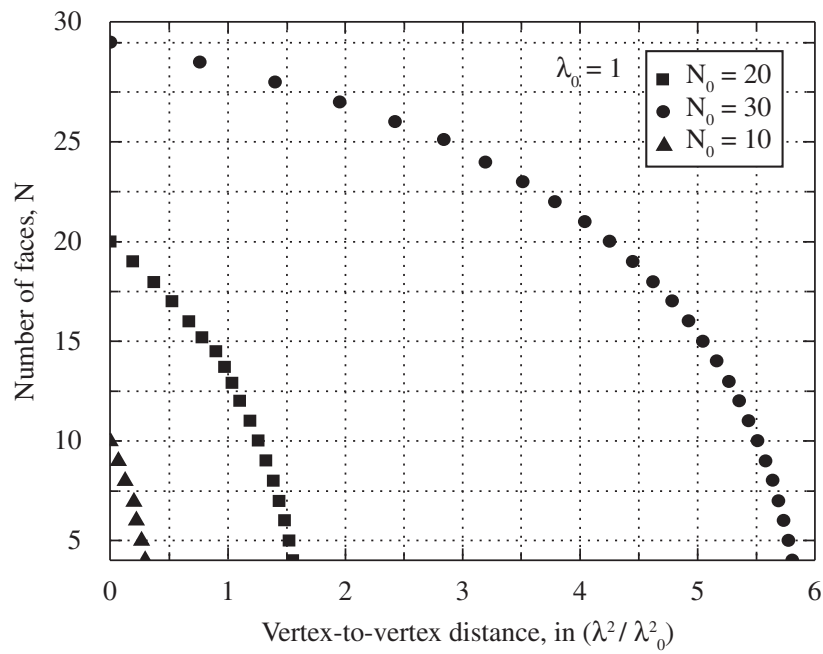

Figure 3. Number of faces of a grain, $N$, as a function of vertex-to-vertex distance, $\lambda$, for different values of the initial number of faces, $N_{0}$. Grains with initially larger number of faces take a much longer time, i.e., larger increase in $\lambda$, to disappear. 
Equation 15 represents the trajectory of a grain, initially with $\mathrm{N}_{0}$ faces, with in $(\mathrm{N}, \mathrm{V})$ space. The trajectories calculated from Equation 15 for $\mathrm{N}_{0}=20$ and $\mathrm{N}_{0}=30$ are shown in Figures $4 \mathrm{a}$ and $\mathrm{b}$, respectively. The grains initially increase their volumes because their boundary curvature is negative, $\mathrm{N}>\mathrm{N}_{\mathrm{C}}=13.397 \ldots$, but lose faces. Their volumes eventually reach a maximum as $\mathrm{N}$ tends to $\mathrm{N}_{\mathrm{C}}$. From then on their boundary curvature changes sign and they start to shrink. Notice that since $\mathrm{dV}>0$ for $\mathrm{N}>\mathrm{N}_{\mathrm{C}}$ and $\mathrm{dV}<0$ for $\mathrm{N}<\mathrm{N}_{\mathrm{C}}, \mathrm{dV}=0$ for $\mathrm{N}=\mathrm{N}_{\mathrm{C}}$ and therefore the maximum volume always occurs at $\mathrm{N}=\mathrm{N}_{\mathrm{C}}$. However the magnitude of the maximum volume depends strongly on $\mathrm{N}_{0}$. As shown in Figures $4 \mathrm{a}$ and $\mathrm{b}$, the grain with $\mathrm{N}_{0}=30$ reaches a much larger maximum volume before it reaches $\mathrm{N}=13.397$ than the grain with $\mathrm{N}_{0}=20$. In this way, even grain with initially concave faces may eventually disappear as required by an overall decrease in number of grains per unit of volume.

\section{Abnormal Grain Growth Kinetics in a Pinned Matrix}

Equation 11 allows the determination of $\mathrm{N}_{\mathrm{A}}$ as a function of the dimensionless time, $\tau=\left(M \gamma / \lambda^{2}\right)$ t. Separating the variables in Equation 11, and integrating from $\tau=0$, when $\mathrm{N}=\mathrm{N}_{\mathrm{A} 0}$, to an AGG time, $\tau$, when $\mathrm{N}=\mathrm{N}_{\mathrm{A}}$, yields the relationship between the number of faces added to the abnormal grain at time $\tau$,

$$
\int_{N_{A 0}}^{N_{A}}\left(\frac{\partial v}{\partial N}\right)_{\lambda} \frac{d N}{(-h-Z \lambda / 2) a}=\int_{0}^{\tau} d \tau^{\prime}=\tau
$$

In order to illustrate this topological aspect of abnormal grain growth we choose as the abnormal grains one initially with $\mathrm{N}_{\mathrm{A} 0}=41$, and another with $\mathrm{N}_{\mathrm{A} 0}=100$ faces. The pinning coefficient is selected to be $-\mathrm{Z} \lambda / 2=\mathrm{h}(40)$, so that abnormal grain growth initiates if $\mathrm{N}_{\mathrm{A} 0}>40$. Note that the value for the mean curvature, $\mathrm{H}$, for a 40 -sided $\mathrm{ANH}$ with $\lambda=1$ is $h(40)=-0.274-$ a value obtained from Equation 2 .

Figure 5 shows the increase in the number of faces, $N_{A}$, of an abnormal grain as a function of dimensionless time, $\tau$, for different initial values of the number of faces on the candidate abnormal grain. The ratio of the spherically equivalent grain radius of the abnormal grain, $R_{A}$, to the spherically equivalent mean grain radius, $R_{M}$, is also plotted as the right-hand ordinate, for comparison.

\section{Conclusions}

A new methodology was introduced to treat normal grain growth. Normal grain growth was analyzed in terms of coupled topological and metrical processes with the help of ANH formalism. We found that for normal grain growth large grains, $N>N_{C}=13.397 \ldots$, increase their volume by increasing their metric component even though they lose faces. On the other hand, smaller grains, $\mathrm{N}<\mathrm{N}_{\mathrm{C}}=13.397 \ldots$, tend to decrease their volume by losing faces even though their metric component increases. For abnormal grain growth in a pinned matrix the metric process is not possible and the abnormal grain grows exclusively by gaining faces, a topological process.

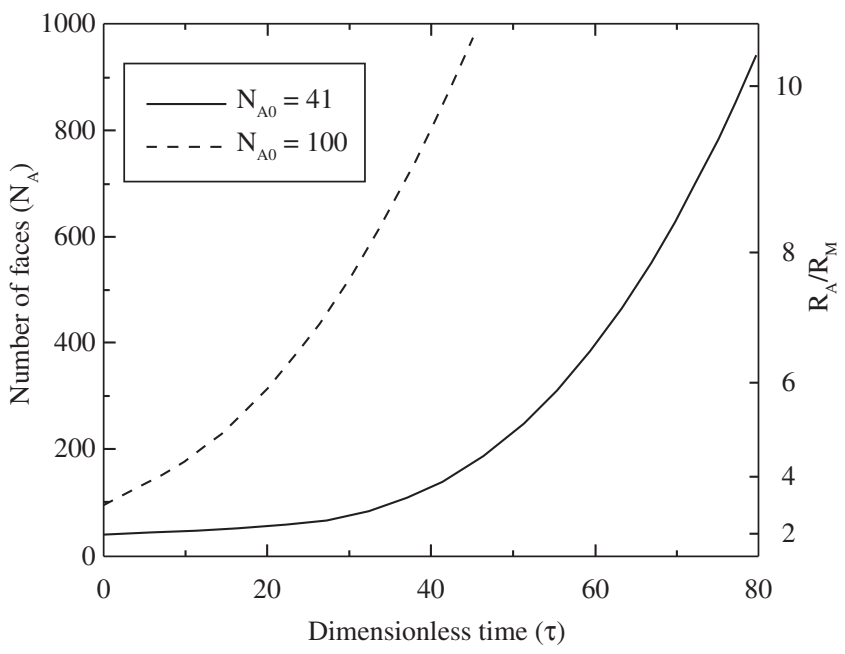

Figure 5. Increase in the number of grain faces, $\mathrm{N}_{\mathrm{A}}$, as a function of dimensionless growth time, $\tau$, for abnormal grains with different initial number of faces, $\mathrm{N}_{\mathrm{A} 0}=41$ and $\mathrm{N}_{\mathrm{A} 0}=100$. Predictions based on Equation 15.

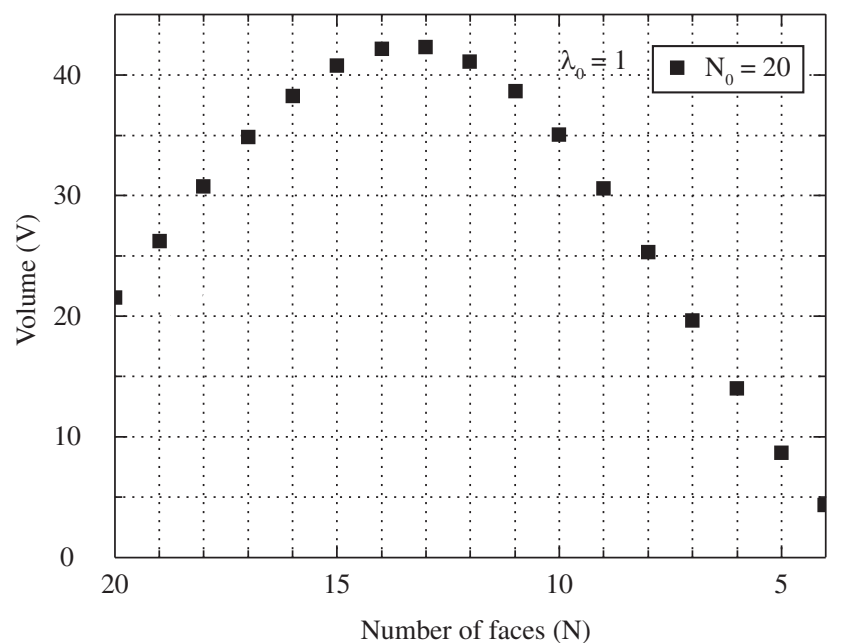

(a)

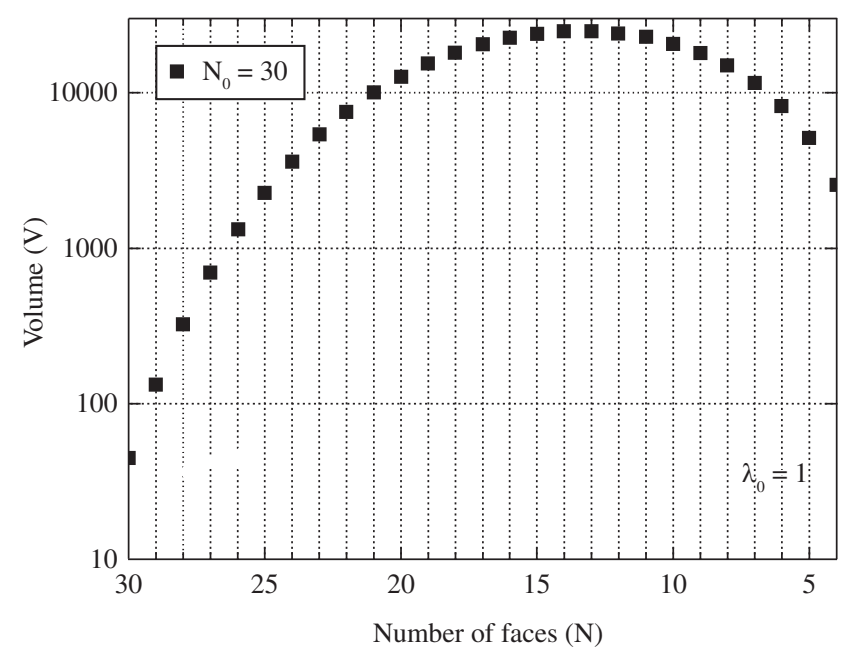

(b)

Figure 4. Grain trajectories in (N,V) space: a) $\mathrm{N}_{0}=20$; and b) $\mathrm{N}_{0}=30$. Grains with initially larger number of faces reach much higher volumes before they start to shrink. 
Thus, by considering the topological details of polyhedral grains, important information is added to our understanding of the principles of normal and abnormal grain growth not provided by previous methodology that often approximated polyhedral grains by a sphere with equivalent volume.

\section{Acknowledgments}

One of the authors (PRR) is grateful to the Conselho Nacional de Desenvolvimento Científico e Tecnológico, $\mathrm{CNPq}$, and to the Fundação de Amparo à Pesquisa do Estado do Rio de Janeiro, FAPERJ, for his financial support.

\section{References}

1. Hillert M. On the theory of normal and abnormal grain growth. Acto Metallurgica. 1965; 13(3):227-238.
2. Glicksman ME. Analysis of 3-D network structures. Philosophical Magazine. 2005; 85(1):3-33.

3. Glicksman ME, Rios PR. Microstructural characteristics of 3-D networks. Zeitschrift für Metallkunde. 2005; 96(10):1099-1105.

4. Rios PR, Glicksman ME. Self-similar evolution of network structures. Acta Materialia. 2006; 54(4):1041-1051.

5. Rios PR, Glicksman ME. Modeling polycrystals with regular polyhedra. Materials Research. 2006; 9(2):231-236.

6. Rios PR, Glicksman ME. Topological theory of abnormal grain growth. Acta Materialia. 2006; 54(19):5313-5321.

7. Rios PR, Glicksman ME. Topological and metrical analysis of normal grain growth in three dimensions. Acta Materialia. 2007; 55(5):1565-1571.

8. Rios PR. Overview no. 62: A theory for grain boundary pinning by particles. Acta Metallurgica. 1987; 35(12):2805-2814. 